\title{
MPC Based Driver's Intention Prediction Method for Vehicle Stability Control
}

\author{
Shunhang Zheng, Bangcheng Zhang, Shaosong $\mathrm{Li}^{*}$, Luping Guo, Guodong Wang, Zheng Li and Xiaohui Lu \\ Changchun University of Technology, Changchun, China \\ ${ }^{*}$ Corresponding author
}

\begin{abstract}
Aiming at improving vehicle handling and stability performance, a model predictive control based vehicle yaw stability controller is designed via active front steering system. In this paper, the reference yaw rate is varied in each prediction horizon, which can predict the driver's intention. And the steering system constraint is also taken into account. The proposed MPC controller is verified on a Carsim simulation platform under the sinusoidal input test maneuver. Simulation results show the benefits of the control methodology used.
\end{abstract}

Keywords- model predictive control; vehicle stability control; active front steering

\section{INTRODUCTION}

Yaw stability control system plays a significant role in vehicle lateral dynamics in order to improve the vehicle handling and stability performances. Active front steering system (AFS) is an effective control system to improve handling stability and active safety performance, and it has been the research hotspots nowadays[1].

Model Predictive Control (MPC) can predict the vehicle dynamics states in the future and has advantage in dealing with multiple control objectives in the case of constraints. [2][3]. Recent contributions to theory and algorithms have enlarged the application spectrum of real-time MPC[4][5]. Several MPC controllers have been proposed for the active front steering control[6][7].

However, in the presented MPC based yaw stability controller the ideal yaw rate is set to be a fixed value in each control cycle, which will cause poor controller performance. In this paper, a variable reference methods is proposed. In each prediction horizon, the reference yaw rate is varied, which can predict the driver's intention.

The organization of this paper is as follows. In Section 2, a vehicle model is built and a control problem is stated in detail. In Section 3, a MPC yaw rate stability controller based on variable reference introduced. Section 4 gives the simulation results to validate the effectiveness of the proposed controller for different cases. The conclusion and future work are summarized in Section 5.

\section{SySTEM MODELING FOR CONTROL DESIGN}

\section{A. Vehicle model}

A 2DOF vehicle model is considered, as shown in Figure 1, which utilizes small angle assumptions and the approximation that the tires on each axle can be lumped together. A constant vehicle longitudinal velocity $U x$ is also assumed. The equations of motion for this model can be written in state-space form as:

$$
\dot{x}(t)=A x(t)+B_{u} u(t)+B_{d} d(t)
$$

where the state variable is yaw rate $\gamma$; the control input is front steering angle $\delta_{f}$; the disturbance inputs is side slip angle $\beta$. The state matrix $A$, input matrix $B_{u}$, disturbance input matrix $B_{d}$ as follows:

$$
\begin{aligned}
& A=\left[\frac{1}{U_{x} I_{z}}\left(l_{f}^{2} C_{f}+l_{r}^{2} C_{r}\right)\right], \\
& B_{u}=\left[\frac{l_{f} C_{f}}{I_{z}}\right], \\
& B_{d}=\left[\frac{1}{I_{z}}\left(l_{f} C_{f}-l_{r} C_{r}\right)\right],
\end{aligned}
$$

where $m$ and $I_{z}$ are the vehicle mass and yaw moment of inertia, respectively; $C_{f}$ is the front tire cornering stiffness; $C_{r}$ is the rear tire cornering stiffness; $l_{f}$ is the distance from C.G. to front axle; $l_{r}$ is the distance from C.G. to rear axle.

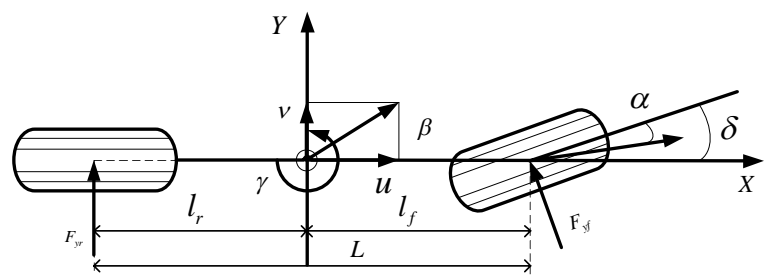

FIGURE I. 2DOF VEHICLE MODEL. 


\section{B. Control Problem Statement}

From the viewpoint of vehicle dynamics, the yaw rate are closely related to vehicle stability, which are controlled by the front steering angle. And the following two objectives are considered:

- good handling and stability.

- smooth steering of vehicle.

In addition, some constraints should be taken into account. Due to actuator saturation, which can be considered as a physical constraint of system. An upper bound on the steering angle magnitude as expressed by the following inequality constraint.

$$
\left|\delta_{f}\right|<\delta_{f \max }
$$

The front wheel steering rate $\Delta \delta_{f}$ is constrained to obtain a smoother control command sequence

$$
\left|\Delta \delta_{f}\right|<\Delta \delta_{f \max }
$$

A 2DOF reference model is designed to determine ideal motion state of vehicle. And the desired yaw response $\gamma_{\text {ref }}$ is derived in [8].

Therefore, on the basis of those discussed above, the stability control problem is described as follows:

- Aiming at keeping yaw stability of vehicle, yaw rate tracks the desired $\gamma_{\text {ref }}$.

- Moreover, for avoiding control saturation and preservation of driver comfort, the front wheel steering angle $\delta_{f}$ and the front wheel steering angle rate $\Delta \delta_{f}$ are constrained.

\section{Design of Model Predictive Controller}

\section{A. Control Scheme}

In this section, a model predictive controller is derived to solve the constrained tracking problem mentioned above. The control scheme of the proposed control system is shown in Figure 2 which contains several subsystems. In the reference model subsystem, the desired value of the vehicle yaw rate is calculated based on steering angle, vehicle longitudinal velocity and tire-road friction coefficient. The input of the vehicle is front wheel steering angle $\delta_{f}$.The output of the vehicle are vehicle yaw rate $\gamma$, vehicle side slip angle $\beta$, vehicle longitudinal velocity $U_{x}$, and tire-road friction coefficient $\mu$.

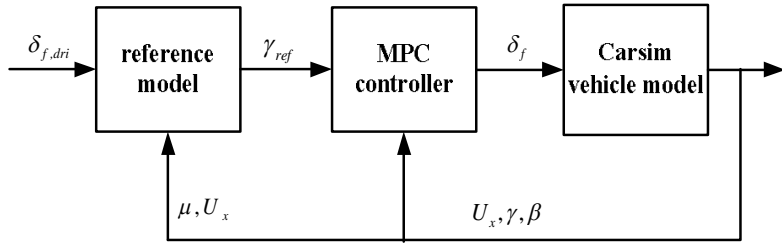

FIGURE II. CONTROL SCHEME.

In order to simplify the design process, the following assumptions are made.

- Carsim vehicle model can accurately represent the real vehicle in this simulation-based study.

- The vehicle parameter values are accurate and constant throughout the simulation, such as vehicle mass.

- All the vehicle state information can be obtained accurately, such as tire-road friction coefficient.

- The vehicle longitudinal speed is maintained constant.

\section{B. Design of the Prediction Equation}

Aiming at solving the tracking problem, the system model (1) is described as a discrete form in (5) with the Euler method. The sample time is $k=\operatorname{int}\left(t / T_{s}\right)$, where $t$ is running time and $T_{s}$ is a fixed step size.

$$
\begin{aligned}
& x(k+1)=A_{c} x(k)+B_{c u} u(k)+B_{c d} d(k), \\
& y(k)=C x(k),
\end{aligned}
$$

Where, $A_{c}=e^{A T_{s}}, B_{c u}=\int_{0}^{T_{s}} e^{A t} d t \cdot B_{u}, B_{c d}=\int_{0}^{T_{s}} e^{A t} d t \cdot B_{d}, C=1$. The discrete form can be written as incremental form in (6) as follows, in which system static error can be eliminated.

$$
\begin{aligned}
& \Delta x(k+1)=A_{c} \Delta x(k)+B_{c u} \Delta u(k)+B_{c d} \Delta d(k), \\
& y(k)=C \Delta x(k)+y(k-1) .
\end{aligned}
$$

For this control system, at sample time $k$, the future control input sequence, $U(k)$ is the optimization vector in the following cost function and the predictive control output sequence is $Y(k)$, which can be defined as follows:

$$
U(k)=\left[\begin{array}{c}
u(k \mid k) \\
u(k+1 \mid k) \\
\mathbf{M} \\
u(k+m-1 \mid k)
\end{array}\right]_{m \times 1}, Y(k)=\left[\begin{array}{c}
y(k+1 \mid k) \\
y(k+2 \mid k) \\
\mathbf{M} \\
y(k+p \mid k)
\end{array}\right]_{p \times 1}
$$

where each vector of $U(k)$ is an array of control inputs $u$, and each vector of $Y(k)$ is an array of system output, $P$ is defined as predictive horizon to extend the forecast of the system future output and $m$ is defined as control horizon. 
The sequence of the control input change, $\Delta U(k)$ is calculated by $\Delta u(k)=u(k)-u(k-1)$, which is equal to zero beyond the control horizon. They are formed as follows:

$$
\Delta U(k)=\left[\begin{array}{c}
\Delta u(k \mid k) \\
\Delta u(k+1 \mid k) \\
\mathrm{M} \\
\Delta u(k+m-1 \mid k)
\end{array}\right]_{m \times 1} .
$$

\section{Driver's Intention Prediction}

Considering that the driver's intention is always changing continuously, the reference yaw rate will be calculated in realtime on the basis of driver's intention. In order to get better controller performance, the reference yaw rate value is set to be variable in predictive horizon.

Here $R(k)$ is defined as output reference sequence. The calculation for driver's intention is formed as follows:

$$
\begin{aligned}
& r(k+1)=r(k)+\lambda *\left|\gamma_{r e f}(k)\right| *\left(\gamma_{r e f}(k)-\gamma_{r e f}(k-1)\right) \\
& r(k+2)=r(k+1)+\lambda *\left|\gamma_{r e f}(k)\right| *\left(\gamma_{r e f}(k)-\gamma_{r e f}(k-1)\right)
\end{aligned}
$$

$\mathrm{M}$

$r(k+P-1)=r(k+P-2)+\lambda *\left|\gamma_{r e f}(k)\right| *\left(\gamma_{r e f}(k)-\gamma_{r e f}(k-1)\right)$,

$$
R(k)=\left[\begin{array}{l}
r(k) \\
r(k+1) \\
\mathrm{M} \\
r(k+p-1)
\end{array}\right]_{p \times 1},
$$

where $\gamma_{\text {ref }}(k)$ is the reference yaw rate from reference model; $\gamma_{\text {ref }}(k-1)$ is the reference yaw rate value at previous sample time, and $\lambda$ is weight coefficient, which can adjust change rate of reference yaw rate, and then affect the tracking performance of vehicle.

Figure 3 describes how the algorithm works in MPC. The red curve is based on the method proposed in this paper. The black curve is based on normal MPC method. It can be seen that the reference value changes in prediction horizon, and the open-loop input is bigger than the normal MPC method. This means a better prediction of future dynamics. The benefits of this algorithm are clear, which can provide more accurate optimization results and better responsiveness.

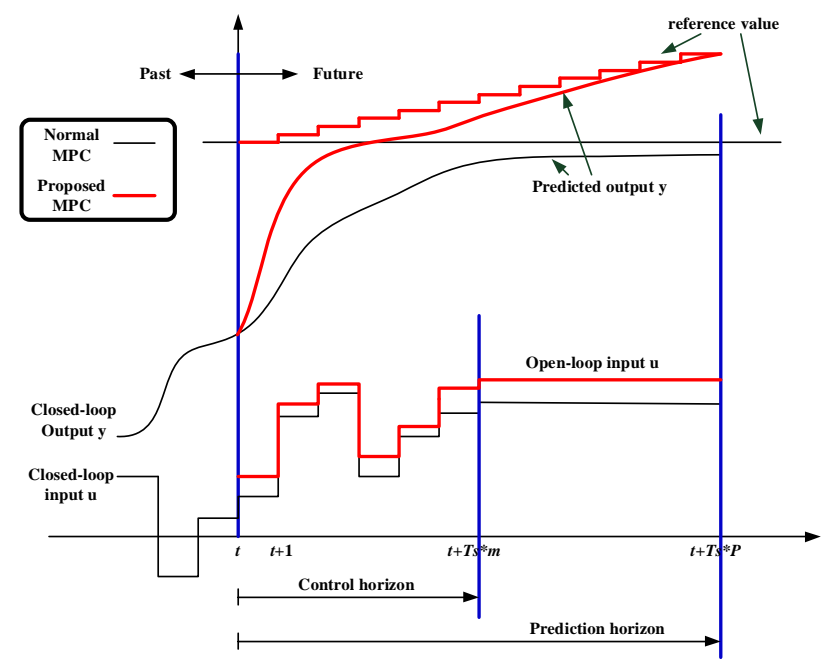

FIGURE III. PREDICTION THEORY

\section{Design of the Optimization Function}

In order to achieve precise yaw rate control, an objective function is designed based on the tracking problem, which is composed of two parts as follows:

(1) The main control requirement is to make actual yaw rate $\gamma$ track the required reference $R(k)$. The criterion for minimizing the deviation between predicted outputs and given references is usually chosen in a quadratic form. Therefore, the first cost function is as follows,

$$
\begin{aligned}
J_{1} & =\|Y(k)-R(k)\|_{Q}^{2} \\
& =\sum_{j=1}^{p}\left[\left(\gamma(k+i \mid k)-\gamma_{r e f}(k)\right)^{2} \cdot Q_{1}\right],
\end{aligned}
$$

where $Q_{1}$ is a positive weight factor for adjusting tracking performance and weight matrix $Q=\operatorname{diag}\left(Q_{1}\right)$.

(2) In order to reduce the change rate of control action and ensure smooth steering, a cost function is formed as follows,

$$
\begin{aligned}
J_{2} & =\|\Delta U(k)\|_{S}^{2} \\
& =\sum_{j=1}^{j=m-1}\left[\left(\Delta \delta_{f}(k+j \mid j)^{2}\right) \cdot S_{1}\right] .
\end{aligned}
$$

Finally, a total objective function of the optimal control problem is defined over a receding horizon consisting of two parts (11)(12), as follows:

$$
\begin{aligned}
J_{m p c} & =J_{1}+J_{2} \\
& =\|Y(k)-R(k)\|_{Q}^{2}+\|\Delta U(k)\|_{S}^{2} .
\end{aligned}
$$


In addition, the constraints in (3)(4) can be handled with by MPC in optimization process at each sample time. The hard constraints of the control input $\delta_{f}$ and $\Delta \delta_{f}$ can be rewritten as follows,

$$
\begin{aligned}
& -\delta_{f \max } \leq \delta_{f}(k+j \mid k) \leq \delta_{f \max }, \\
& -\Delta \delta_{f \max } \leq \Delta \delta_{f}(k+j \mid k) \leq \Delta \delta_{f \max }, \\
& j=1,2 \ldots, m-1 .
\end{aligned}
$$

\section{E. Model Predictive Control Law}

The linear optimization problem is solved by using a quadratic programming $(\mathrm{QP})$ method[9], which can be defined as follows:

$$
\min _{x} x^{T} H x-g^{T} x
$$

$$
E x<b,
$$

where $H$ is symmetric matrix; $g$ Gradient vector, $E$ and $b$ are constraint matrix. According to MPC control strategy, the first element of the $U(k)$ is implemented and the calculation is repeated at each time instant. Hence, at time instant $k$, the resulting state feedback control law is as follows,

$$
u(k)=[1,0, \ldots, 0] \cdot U(k)
$$

where $u(k)$ is a set of optimal vectors for active steering angle.

\section{SIMULATION COMPARISON AND ANALYSIS}

In this section, simulation results are shown to demonstrate the validity of the proposed MPC controller compared with normal MPC controller. The simulation is evaluated on Carsim vehicle model. The control effects of the designed MPC controller on sinusoidal input test maneuver at high speed is verified. The simulation conditions are as follows: The vehicle speed is $120 \mathrm{~km} / \mathrm{h}$, The road surface is dry concrete with tireroad friction coefficient as 0.85 and driver input is the sinusoidal input.

Simulation results of vehicle yaw rate is shown in Figure 4(a). It can be seen that the normal MPC controller have a bigger tracking error than the proposed MPC controller based on variable reference yaw rate, which means that the driver's intentions method has good performance. And Figure 4(b) describes the vehicle front wheel angle at sinusoidal input maneuver. The experiment result showed that proposed MPC controller has faster response than the normal MPC controller.

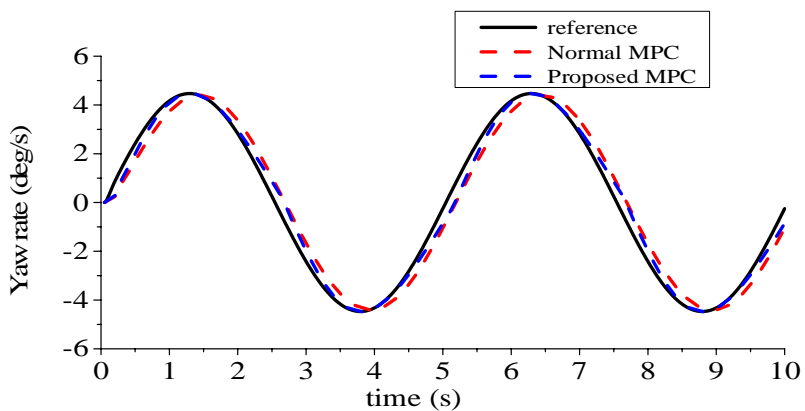

(a) yaw rate

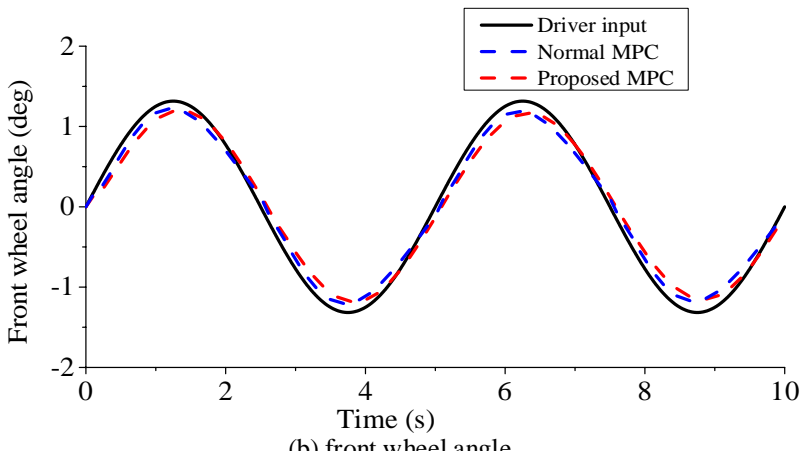

(b) front wheel angle

FIGURE IV. SIMULATION RESULTS OF SINUSOIDAL INPUT TEST MANEUVER WHEN M $=0.85$.

\section{CONCLUSIONS}

This paper focuses on yaw stability control via active front steering, and presents a driver's intention prediction method The reference yaw rate value is varied in prediction horizon, which means that the proposed MPC controller has better control accuracy. The proposed MPC controller is verified on an Carsim simulation platform under the sinusoidal input test maneuver at high speed. Simulation results show the benefits of the control methodology used.

In the future, effectiveness of the control strategy will be validated in the currently being built vehicle test bench. In order to achieve better control performance, further study about the proposed MPC controller is needed.

\section{ACKNOWLEDGMENT}

The work is supported by the Jilin Province Science and Technology Found for Young Scholars (No.20170520097JH; No.20160520106JH), and by the Key Technology and Development Program of Jilin Province (No.20170201005GX).

\section{REFERENCES}

[1] Jin X J, Yin G, Li Y, Li J. "Stabilizing Vehicle Lateral Dynamics with Considerations of State Delay of AFS for Electric Vehicles via Robust Gain - Scheduling Control," Asian Journal of Control, 2016.

[2] Jiechao Liu, Paramsothy Jayakumar, Jeffrey L. Stein \& Tulga Ersal. “A study on model fidelity for model predictive control-based obstacle avoidance in high-speed autonomous ground vehicles," Vehicle Dynamics Control, vol. 54 no. 11 pp. 1629-1650, August 2016. 
[3] Németh B, Gáspár P, Péni T. "Nonlinear analysis of vehicle control actuations based on controlled invariant sets," International Journal of Applied Mathematics \& Computer Science, 2016, pp. 31-43.

[4] Choi M, Choi S B. "MPC for vehicle lateral stability via differential braking and active front steering considering practical aspects," Proceedings of the Institution of Mechanical Engineers Part D Journal of Automobile Engineering, 2016.

[5] Qu T, Chen H, Cao D, H Guo, B gao. "Switching-Based Stochastic Model Predictive Control Approach for Modeling Driver Steering Skill," IEEE Transactions on Intelligent Transportation Systems, 2015, pp. 365375.

[6] Beal C E, Gerdes J C. "Model Predictive Control for Vehicle Stabilization at the Limits of Handling," IEEE Transactions on Control Systems Technology, vol. 21 no. 4 pp. 1258-1269, July 2013.

[7] Yakub F, Mori Y. "Enhancing the stability of unmanned ground sport utility vehicles through coordinated control under MU-split and gust of wind," Journal of Engineering Science \& Technology, 2016, pp. 14501469.

[8] Bingtao Ren, Hong Chen, Haiyan Zhao, Lei Yuan. "MPC-based yaw stability control in in-wheel-motored EV via active front steering and motor torque distribution," Mechatronics, vol. 38 pp. 103-114, 2016.

[9] Maciejowski J M. "Predictive control with constraints," Prentice Hall, 2011 